\title{
Análisis del funcionamiento y rol de los Grupos de Acogimiento en la atención de la salud mental comunitaria. Lima, Perú, 2019.
}

Analysis of functioning and role of Foster Groups in community mental health care. Lima, Peru, 2019.

\author{
Humberto Castillo-Martell 1,a, Hugo Aguirre-Castañeda ${ }^{2, b}$, Fernando Carbone-Campoverde ${ }^{3, c}$, Rocio \\ Valverde-Aliaga ${ }^{4, d}$, Rafael Sepúlveda-Jara ${ }^{5, e}$, Alberto Fernandez-Liria ${ }^{6, f}$
}

\section{RESUMEN}

Objetivo: Analizar el funcionamiento y discutir el rol de un modelo participativo de formulación diagnóstica y terapéutica denominado Grupos de Acogimiento (GDA), implementados en un Centro de Salud Mental Comunitario (CSMC) en Perú. Material y métodos: La investigación se basó en la observación participante de expertos en salud mental comunitaria, salud pública, ciencias de la comunicación y ciencias sociales, en un total de 24 sesiones de GDA realizadas en el CSMC Nancy Reyes de Chorrillos, Lima, Perú, durante los años 2018-2019. Resultados: Los GDA están estructurados en fases que siguen un proceso lógico de formulación de diagnósticos y planes de manejo que, al llevarse a cabo de forma concurrente con la participación de profesionales de diferentes disciplinas y dirigido a un pequeño grupo de usuarios, permite que tanto los diagnósticos como los planes tengan un carácter más integral. Se va generando un ambiente de confianza que permite que las personas compartan experiencias de sufrimiento e intimidad en niveles que con frecuencia superan los expresados en ámbitos de la tradicional relación profesionalusuario. El diálogo interdisciplinario es permanente y los usuarios alternan roles de analizados y analizantes, dando señales de un empoderamiento progresivo. Conclusiones: Los GDA operacionalizan los principios fundamentales de la salud mental comunitaria, son una herramienta valiosa para la expansión del Modelo de Cuidado Integral como eje de la política pública de salud en el Perú, diluyen las barreras comunicacionales propias de la relación profesional-usuario y acortan las distancias sociales de los participantes, posibilitando mejores alianzas terapéuticas y de cuidado.

PALABRAS CLAVE: Salud mental comunitaria, diagnóstico en salud mental, diagnóstico psiquiátrico.

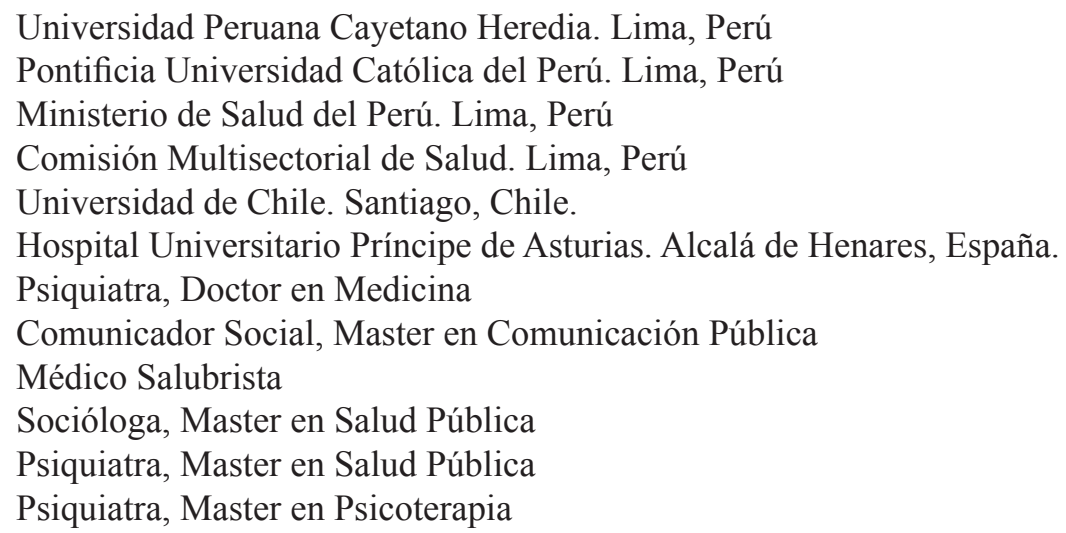




\section{SUMMARY}

Objective: The operationalization and the role of a participatory model of diagnostic and therapeutic formulations called Foster Groups, implemented in a Community Mental Health Center in Lima, Peru, are analyzed. Material and Methods: The research was based on the participanting observation by experts in community mental health, public health, communication and social sciences, of a total of 24 Foster Group sessions held at the Nancy Reyes Community Mental Health Center in Chorrillos, Lima Peru, during the years 2018-2019. Results: The Foster Groups are structured in sequential phases that follow a logical process of diagnostic formulations and management plans, concurrently performed by professionals from different disciplines for a small group of users, thus allowing both, diagnoses as plans, to be more comprehensive. An environment of trust is generated, allowing people to share experiences of suffering and intimacy at levels that often exceed those expressed in the traditional doctor-patient relationship. Interdisciplinary dialogue is permanent and users alternate roles of analysands and analysers, showing signs of progressive empowerment. Conclusions: Foster Groups operationalize the fundamental principles of Community Mental Health, are a valuable tool for the expansion of the Comprehensive Care Model as the axis of public health policy in Peru, soften the communication barriers of the doctor-patient relationship, and reduce the social distances between participants, enabling better care and therapeutic alliances.

KEYWORDS: Community mental health, mental health diagnosis, psychiatric diagnosis.

\section{INTRODUCCIÓN}

El diagnóstico en salud mental, y en la medicina en general, es muy relevante en el proceso de atención. Reconocer problemas de salud mental detrás del sufrimiento y las conductas problemáticas y comprender sus posibles causas -manifiestas y ocultases fundamental para seleccionar las intervenciones a implementar a fin de lograr el objetivo de mejorar la salud mental de personas, familias y/o comunidades (1). Al ser la salud-enfermedad mental un fenómeno altamente complejo es objeto de múltiples disciplinas del campo de la salud y fuera de él. El diagnóstico de la problemática de salud mental de una persona en concreto puede ser distinto dependiendo de la disciplina desde la cual se analiza. Generalmente las diferentes perspectivas se complementan y enriquecen, pero también pueden contradecirse unas con otras. Precisamente por eso, contribuyen a una visión más amplia de la complejidad del objeto, lo cual hace a la interdisciplinariedad y a la articulación de las diferentes formas de analizar, explicar y comprender el proceso salud-enfermedad mental, una práctica valiosa para la atención y cuidados (2).

El diagnóstico psiquiátrico pone énfasis en las mediaciones biológicas de los problemas de salud mental y los tipifica como enfermedades o trastornos (3). Los diagnósticos psicológicos y psicoterapéuticos están centrados en reconocer y explicar emociones, pensamientos y otras funciones mentales asociadas a sufrimiento y desadaptación basándose en diversas teorías sobre el comportamiento humano (4). Los diagnósticos de enfermería se refieren principalmente a la identificación de necesidades de atención y cuidado de las personas inmersas en algún problema de salud mental (5), mientras que los diagnósticos de trabajo social se enfocan en las condiciones de vida de las personas y cómo estas influyen en su proceso salud-enfermedad-atención. Asimismo, es importante resaltar que las emociones y conductas de los seres humanos, son objeto de reflexión e intervención desde la educación, la religión, la política, la moral, la seguridad pública y otras prácticas sociales, con narrativas que son relevantes a la hora del diagnóstico y las intervenciones desde las diferentes disciplinas de la salud mental (6).

El problema o los riesgos de los diagnósticos convencionales o unidisciplinares en salud mental, derivan principalmente del reduccionismo epistemológico, sea "biologista" o "psicologista", y de la asimetría de poder en la relación profesionalusuario, donde la voz oficial para el análisis y diagnóstico está ostentada únicamente por el profesional (7). Desde el inicio de la interacción y de forma tácita, se plantea una diferencia sustantiva y radical entre usuario y profesional, donde el primero se presenta como quien tiene problemas y el segundo, como quien tiene las soluciones. La supuesta superioridad del "conocimiento científico" sobre el "conocimiento común" aceptado socialmente, se hace extensiva a todas formas de saber del profesional y usuario participantes en el acto de diagnóstico, de tal manera que se establece una una jerarquía en la validez de las observaciones, experiencias, interpretaciones y análisis de uno y otro. A esta relación jerárquica y de dominación-subordinación de saberes, que se extiende 
de lo individual a lo colectivo, Boaventura de Sousa Santos la denomina "epistemicidio" (8). En "su" consultorio privado o público, el profesional controla el escenario, el tiempo, los turnos de hablar, es él quien hace las preguntas, y quien saca las conclusiones, define el diagnóstico y hace las indicaciones, estableciendo y consolidando su hegemonía sobre el proceso de diagnóstico y tratamiento (9).

Es por estas razones que los enfoques modernos de Salud Mental Comunitaria (SMC) cuestionan las formas unidisciplinares y reduccionistas de los diagnósticos, y propician formas más comprehensivas, integrativas, interdisciplinarias y participativas que den cuenta de la complejidad (biológica, psicológica y social) de los fenómenos de la salud mental $(10,11)$. Con ese fin, se fomentan las prácticas colaborativas entre los actores múltiples y diversos en todos los procesos de atención y cuidado de la salud mental de individuos y colectivos. Esto significa superar los límites de la epistemología y la objetividad positivista centrada en la evidencia empírica y abrir paso a aproximaciones epistemológicas, donde la intersubjetividad y los múltiples puntos de vista son la base de la "objetividad diagnóstica" (12). Sin embargo, la articulación o integración de los diagnósticos de cada disciplina suele ser un proceso complejo, complicado y costoso que requiere del esfuerzo consciente, colectivo y sostenido de todos los involucrados, por lo que esta práctica suele quedarse en el ámbito declarativo, abandonarse con el correr del tiempo o realizarse sólo en casos excepcionales.

Los Centros de Salud Mental Comunitarios (CSMC) que se están implementando en Perú (13), como parte de una reforma reciente que recoge experiencias latinoamericanas y europeas, posibilitan el desarrollo de experiencias innovadoras que, siguiendo los principios de la SMC, permiten en forma colaborativa con los usuarios y comunidades encontrar las mejores formas de actuar en esta compleja y abundante problemática de la salud mental (14). Es así que en el CSMC Nancy Reyes de Chorrillos, se implementó un modelo de diagnósticos interdisciplinarios simultáneos en pequeños grupos de usuarios, denominado Grupos de Acogimiento (15) como alternativa a las convencionales entrevistas o consultas individuales y secuenciales en la formulación de diagnósticos y planes iniciales de cuidados en salud mental.

Los Grupos de Acogimiento consisten en una sesión semi estructurada de aproximadamente dos horas de duración donde los profesionales involucrados en la atención (enfermeros, psicólogos, psiquiatras y otros, según disponibilidad) entrevistan en forma simultánea, con un grupo de 3 a 6 usuarios -y sus acompañantes de ser el caso- que buscan atención en el establecimiento de salud por primera vez y realizan colaborativamente la tarea de formulación de diagnósticos y planes de cuidado para cada uno de los usuarios. La sesión se desarrolla en cuatro momentos semi estructurados: Presentación horizontal de participantes, comprensión empática mutua, exploración colectiva de causas y decisiones terapéuticas compartidas. Cada sesión tiene un objetivo y diversas estrategias y herramientas para lograrlo. La sesión es conducida por un moderador con la posibilidad de alternancia de este rol entre varios profesionales.

Así, la pregunta principal en esta investigación fue: ¿Hasta qué punto los Grupos de Acogimiento pueden constituirse en una alternativa válida y viable para la formulación de diagnósticos y planes iniciales de cuidado a ser aplicada cotidianamente en los Centros de Salud Mental Comunitaria y otros ámbitos de la atención de salud mental? Con el fin de responder esta pregunta, el objetivo es analizar el funcionamiento y discutir el rol de los Grupos de Acogimiento, en la atención de la salud mental, desde las perspectivas de la salud comunitaria, la comunicación social, las ciencias sociales y la salud pública.

\section{MATERIAL Y MÉTODOS}

La investigación se realizó en base a la observación participante por expertos en salud mental comunitaria, salud pública, ciencias de la comunicación y ciencias sociales externos al equipo de trabajo. En un total de 24 sesiones de Grupos de Acogimiento realizadas en el CSMCNancy Reyes de Chorrillos, Lima(Perú), durante los años 2018-2019, se contó con la presencia de al menos uno de los observadores, que hacían sus propias anotaciones. Las observaciones se complementaron con breves entrevistas no estructuradas individuales y grupales a usuarios participantes; y con grupos de discusión con profesionales que participaron en las sesiones, que enriquecieron las anotaciones de los observadores (no fueron procesadas con con técnicas cualitativas específicas). Cabe mencionar que fueron los propios autores los que hicieron la observación participante, y uno de ellos en varias ocasiones fue moderador del grupo observado, aportando al análisis desde una perspectiva auto etnográfica. 


\section{RESULTADOS}

\section{Sobre el proceso de formulación de diagnósticos y planes terapéuticos}

Se observa que las sesiones siguen un patrón bien estructurado de fases donde cada una de ellas está bien delimitada. Los participantes se suelen involucrar en la misión de cada fase lo cual se pone de manifiesto en las tonalidades de la voz y la dinámica diferente de las intervenciones en cada una de ellas. Las fases siguen el proceso lógico de las ciencias de la salud para la construcción de diagnósticos y planes de acción: Presentación horizontal de participantes, comprensión empática mutua, exploración colectiva de causas, y decisiones terapéuticas compartidas. Se observa que el éxito en cada fase condiciona el éxito en la siguiente. La presentación crea la confianza para que los usuarios puedan narrar sus problemáticas, de una manera suficientemente profunda como para poder identificar sus síntomas y condiciones mentales, en base a las cuales, en las fases siguientes, se formulan casos, hipótesis e incluso diagnósticos de trastornos mentales clasificados en los sistemas de CIE 10 o DSM 4-5.

"La psiquiatría fenomenológica tiene pasión por poner etiquetas [diagnósticos] con poca relevancia para la toma de decisiones iniciales de cuidado. Los denominados diagnósticos comunitarios a los quesellega en los grupos de acogimiento, alseparar los problemas asociados a expectativas insatisfechas de los padres sobre los comportamiento sus hijos, de aquellos que son el resultado de las circunstancias adversas y de las que puede reflejar un trastorno en la estructura emocional de la persona, y dentro de estos los tienen un carácter ansioso depresivo o de mayor complejidad, son suficientes para tomar decisiones iniciales de tratamiento y cuidado. Lo que se pierde en precisión, se gana en consenso y aceptación". (Psiquiatra experto en salud mental comunitaria).

El hecho de que este proceso se haga de forma concurrente por diversos profesionales en el mismo acto y con la participación activa de los usuarios lo hace más eficiente y es una mejora frente a los procedimientos convencionales. En primer lugar, porque se obtiene la información necesaria; en segundo lugar, porque permite elaborar una formulación compartida del problema, "co construida entre el paciente, sus compañeros y el equipo terapéutico"; en tercer lugar, porque permite distinguir los niveles de cuidados requeridos por el paciente; y en cuarto lugar, porque minimiza el riesgo de etiquetar y estigmatizar:

"En el grupo tuvimos una persona con sintomas obsesivos, otra con alucinaciones, otra con sintomas de depresión, otra con conductas de cortarse, otra con retardo del desarrollo y otra que tenía un problema de violencia con el padre. Realmente una diversidad y nadie fue menoscabado en su posibilidad de expresar y probablemente se haya dado un beneficio a las personas tanto por las similitudes como por las diferencias". (Psiquiatra experto en salud mental comunitaria).

Se observa también que cada profesional en la sesión cumple con los objetivos que buscaría en una entrevista individual, con la ventaja de tener la perspectiva de las otras disciplinas en el mismo acto. También es evidente que cada usuario cumple con el objetivo de expresar su problemática y recibir interpretaciones y propuestas para recuperar o mejorar su salud mental, con la ventaja de haber sido espectador y protagonista de situaciones en la que él o ella pueden verse reflejados en las situaciones de los otros.

"Los diagnósticos de aproximación individuales se dieron en esta sola sesión inicial, de forma muy precisa, multidisciplinaria e integral. El proceso de sanación terapéutica también se inició en esta sesión inicial; los usuarios descubrieron aspectos de resiliencia que no pensaban que aún fueran portadores. En un sistema hospitalario institucionalizado esto hubiera tomado posiblemente entre 4 a 6 sesiones distanciadas en el tiempo". (Médico salubrista).

De esta manera, los Grupos de Acogimiento, estructurados en fases secuenciales siguiendo un proceso lógico de formulación de diagnósticos y planes de intervención (conjunto de intervenciones, biologicas, psicologicas y sociales), pero de forma concurrente para un pequeño grupo de usuarios y con la participación de profesionales de diferentes disciplinas, permite que tanto los diagnósticos como planes sean más integrales.

\section{Sobre la construcción de confianza y confidencialidad}

En las sesiones de los Grupos de Acogimiento se observó que desde el inicio se va creando un ambiente de confianza y confidencialidad. Esto se logra a través de múltiples mecanismos, entre ellos, la mutua retroalimentación de la confianza y la fuerza de la 
Análisis del funcionamiento y rol de los Grupos de Acogimiento en la atención de la salud mental comunitaria. Lima, Perú.

escucha colectiva y empática para activar relatos de contenido emocional:

"Cuando se da inicio a la dinámica de acogimiento, el facilitador o facilitadora designada abre la sesión con una fórmula de entrada propia de las narraciones orales y cuentos populares. Esta fórmula confiere al momento una trascendencia comunicativa importante pues le sigue la pregunta acerca de: ¿Cuál es el sufrimiento que los trae aqui al CSMC?; y como un acto de magia narrativa, se abre la sesión de acogimiento y empieza a fluir la comunicación". (Comunicador social).

"Mediante el turno de presentaciones indistintamente de profesionales y a los pacientes mencionando solo nombre (sin apellido), personas conlasqueconvive, lugardenacimiento, actividades de esparcimiento y otras variables personales se crea elementos de identificación entre los participantes sin la distinción central y abismal de médicos y pacientes, cambiando radicalmente el régimen de comunicación y sosteniendo una horizontalidad a lo largo de todo el proceso". (Comunicador social).

Los usuarios que han perdido confianza en sus propias interpretaciones sobre sus problemas, construyen nuevas interpretaciones en la interacción con sus pares y profesionales. El relato de los demás parece ayudar a esclarecer sus propias narrativas. Muchos elementos expresados por unos son piezas claves en narrativas de otros. Asimismo, es importante la alternancia de roles, en la sesión de grupo comparten su historia con los demás usuarios pero también observan a quienes cuentan su historia, preguntando y proponiendo explicaciones y alternativas de solución. Esta práctica refuerza la comprensión y modifica la narrativa de sus propias problemáticas.

La sensación que tienen los usuarios de que varias personas están genuinamente interesadas en escuchar su problemática, les genera confianza y los anima a expresar sus vivencias más íntimas. Esto genera un círculo virtuoso de confianza y expresión auténtica del sufrimiento. Del mismo modo, la presencia de pares, es decir, de otros usuarios que también se encuentran buscando ayuda, contribuye en la generación de seguridad para expresar y compartir sus vivencias personales, que como mencionaron algunos pacientes, no habrían podido expresar en entrevistas o consultas tradicionales:
"Me ha llamado la atención que rápidamente se ha generado la confianza para expresar cuestiones intimas en este espacio público. Las personas han expresado elementos dolorosos de historias de familia con pares y parejas. En ese proceso varias lágrimas han caído en medio de una adecuada contención. He observado el compromiso muy profundo de los padres y los adolescentes por contar su situación: una madre asumiendo responsabilidad en la génesis del problema de hijo, un adolescente con aparente retraso del desarrollo cognitivo expresando con mucha claridad lo que le sucede y lo que a su parecer le sucede a los otros adolescentes. Probablemente lo que mueve a expresar tantas cosas intimas es la atención con la que las otras personas escuchan. Esto lo dijo claramente una persona diciendo "nunca me he sentido tan escuchada como ahora". Es estimulante ver cómo las personas dialogan, cómo se mueven, cómo conversan, y cómo salen y entran en diferentes roles a diferencia de las relaciones ипо а иno". (Comunicador social).

La expresión de las experiencias íntimas de cada usuario, genera automáticamente un sentido de confianza, estableciéndose un pacto implícito de confidencialidad, el cual refuerza e incluso hace poco necesario el pacto explícito que se da al inicio de la sesión. Este es un elemento importante de las observaciones de esta investigación ya que suele considerarse que el pacto de confidencialidad se genera entre dos personas, lo cual probablemente sea una expresión de prácticas confesionales presentes en nuestras culturas:

"Estoy sorprendido, porque en el mundo de la comunicación, la intimidad es dificilmente comunicable. Creo que es una virtud de esta metodología lograr que las personas hablen sobre todo en una primera sesión y con tantas personas todavía extrañas. La condición para comunicar se va generando por la dinámica, el espacio, los tonos comunicacionales, etc. Hay como una secuencia que hace que la información que se pone sobre la mesa sea cada vez más importante, más tensa y más reveladora. Se observan dos niveles de involucramiento: uno para contar lo suyo yotro para escuchar al otro que se retroalimentan mutuamente. Si él puede hablar, yo también. La dinámica es amable y anima a participar". (Comunicador social). 
Asimismo, es importante en este proceso de compartir problemáticas, entender las diversas formas en las que se comunican las personas y su rol en la dinámica de grupo. En este sentido, es importante leer e interpretar correctamente lo que dicen a través de gestos o mediante el uso de distintas palabras, es decir, es importante prestar atención a las formas de comunicación no verbales y no tan explícitas:

"Las personas van calibrando qué, cuánto y cómo expresarse, en lo cualjuega mucho la comunicación gestual. Todos se están leyendo unos a otros y en una amplia gama de oyentes, es altamente probable encontrar a uno más que gestualmente alienta y anima a compartir más de su subjetividad. Por ejemplo, Alberto fue reservado pero dijo las palabras claves y anunció las pistas: predisposición al trago, al uso de drogas, etc. No es que no lo dijo, lo dijo a su manera. (Comunicador social).

Se evidencia que la confidencialidad de grupo es resultante de la propia dinámica y protege de modo suficiente la intimidad de las personas participantes, lo cual se corrobora con la ausencia de reportes sobre problemas en los grupos durante el largo periodo de observación:

"El único riesgo que se me ocurre si pienso en extenderlo, por ejemplo, a la práctica en mi país tendría que ver con el tema de la confidencialidad sobre el que en este momento hay mucha sensibilidad. Es cierto que el encuadre inicial resalta el principio de confidencialidad, pero esto podría no ser considerado suficiente por nuestros comités de ética. En cualquier caso, esto plantea una cuestión problemática que habría que estudiar cómo se resuelve, pero no es un impedimento. (Psiquiatra, experto en salud mental comunitaria).

De esta manera, la dinámica de los Grupos de Acogimiento progresivamente va generando un ambiente de confianza que permite que las personas compartan experiencias de sufrimiento e intimidad en niveles que con frecuencia superan los expresados en ámbitos de la relación médico paciente tradicional.

\section{Sobre las relaciones de poder y la articulación de saberes disciplinarios y comunitarios.}

En la dinámica destaca el rol del moderador que puede ser psiquiatra, psicólogo, enfermera u otro profesional que modera los turnos y condiciones del habla siguiendo la estructura de fases o momentos estandarizados. En general, se observa la tendencia y esfuerzo permanente de moderadores a horizontalizar las relaciones y limitar los excesos, abusos y desequilibrios de poder:

"El poder en la sesión se ejerce al establecer las reglas del grupo, cuando se otorga la palabra, se pregunta, se interrumpe, se define los problemas, se respalda unas explicaciones y no otras, se parafrasea, se da indicaciones, se aconseja, se critica, se corrige. Si bien estas acciones están más concentradas en el moderador y los profesionales, también son ejercidas por los usuarios. Que el doctor inicie y ponga las condiciones del diálogo es una condición previa latente, esperada y de hecho el poder profesional no desaparecerá pero hay esfuerzo para que las brechas en la relación de poder se minimicen. La brecha de poder aparece en el momento que debe aparecer. Ocurre en el momento que debe estar, lo suficiente y por el momento oportuno". (Socióloga experta en salud pública).

Se observa que las brechas de poder que devienen de la heterogeneidad de roles e identidades sociales de los participantes (profesionales y usuarios) va cambiando y desdibujándose en los diferentes momentos de la sesión, y es quizás este, el aporte más valioso de Los Grupos de Acogimiento en relación a las formas convencionales de terapia donde la brecha entre el profesional y el usuario es constante y con frecuencia se refuerza y acrecienta a lo largo del proceso. Entre los profesionales se observa diferencias en el nivel de participación, unos hablan más que otros. Preguntan, ofrecen interpretaciones y argumentan posiciones con diferentes niveles de asertividad y receptividad entre los participantes. Se observa una cierta competencia entre ellos sin embargo no necesariamente sigue una línea jerárquica biomédica tradicional. Se observa el esfuerzo en las narrativas profesionales para tomar en cuenta y complementar otras perspectivas. En este sentido, se observa cómo los profesionales de unas disciplinas van matizando sus narrativas con categorías típicas de otras disciplinas. Así, parece que los grupos crean una pauta para tratar de incluir las más variadas explicaciones, encontrando un momento de síntesis en los denominados diagnósticos comunitarios.

"Me costó trabajo identificar las profesiones de los profesionales participantes. En algún momento 
Análisis del funcionamiento y rol de los Grupos de Acogimiento en la atención de la salud mental comunitaria. Lima, Perú.

el psicólogo hablaba como psiquiatra, la enfermera como psicólogo y el psiquiatra como trabajador social ....". (Socióloga experta en salud pública).

Entre los usuarios también se observan relaciones de poder. En primer lugar, al interior de las familias, es decir, entre padres e hijos o entre los adultos y sus acompañantes. Se observa un empoderamiento entre pares, especialmente en los casos de niños, adolescentes y de mujeres afectadas por violencia.

"Los adolescentes con distintas habilidades comienzan a elaborar su discurso sobre el problema, algunos con mucha disposición y facilidad, otros con dificultades. Ningún adolescente calla, todos participan, algunos son críticos y algunos interactúan entre sí con preguntas, impresiones y hasta reclamos o consejos. En la sesión, un adolescente confrontó abiertamente a su madre diciendo que él, al inicio de la sesión, pensaba que todo lo que le pasaba era por su culpa y es que no se había dado cuenta que su familia tenía más problemas que la de los otros chicos del grupo y que eran ellos los que necesitaban más ayuda". (Socióloga experta en salud pública).

Del mismo modo, se manifiestan diferencias en las condiciones y estatus sociales entre los usuarios que llevan a poner en discurso una diversidad de propuestas explicativas provenientes de diferentes experiencias, enfoques y cosmovisiones. Si bien las propuestas provienen principalmente de profesionales, las propuestas de los usuarios siempre están presentes y fuerzan a los profesionales a incluirlas o por lo menos discutirlas en sus narrativas. La dinámica de la sesión tiende a atenuar y hacer converger las diferencias. En este sentido, las relaciones de poder al interior están dispersas en varios ejes: profesional, social, etario, cultural, etc; relativizando el eje Médico (u otro profesional)-Paciente, en el que se concentra la brecha de poder cuando la relación es uno a uno.

"En el grupo había una diversidad de clases o procedencias sociales: Gregorio es un hombre andino; Alberto, cajamarquino migrante de retorno de Argentina; Juana y Jorge, limeños, uno más popular y otro más clase media. También habia diferencias en los niveles intelectuales y de elocuencia, incluso en el grupo había una persona con cierto retardo mental, con lo que el grupo podría haber perdido funcionalidad, sin embargo no fue así. Todos estuvieron involucrados y las relaciones jerárquicas sociales se atenuaron. Se puso el piso parejo y se emparejaron los egos y las jerarquías sociales. Entre los profesionales también se observó heterogeneidades sociales culturales. Más diferencias culturales que económicas y procedencia de diferentes regiones del país y de diferentes distritos de la ciudad de Lima. También heterogeneidad etaria y experiencia profesional". (Comunicador social).

También ocurre que en algunos momentos surgen formas de lucha por el poder e incluso intentos de tomar el control de la sesión; sin embargo, en general estos episodios son controlados por las propias estructuras para participar definidas por el moderador $\mathrm{u}$ otros profesionales.

"En la sesión hubo un intento de competir con la hegemonía. La señora Juana, si no la cortaban se agarraba la conversación con el argumento de que ella va ayudar y salvar el mundo. No sintió que la estaba callando pero que sí había un régimen de alternancias para hablar que requiere cierta autorización o venia de los profesionales". (Comunicador social).

\section{Sobre la funcionalidad y eficiencia}

Los Grupos de Acogimiento en un periodo de 2 horas permiten el diagnóstico interdisciplinario, la formulación de planes iniciales de intervención y adicionalmente, una experiencia terapéutica y educativa de un promedio de 4 a 5 personas. En el modelo convencional de consultas individuales y secuenciales, tomaría al menos el triple de horas/ profesional y el triple de horas/paciente.

"Cada uno de los 6 usuarios recibió en términos prácticos 2 horas de cuidado inicial integral. La suma de las intervenciones individuales les ayudó a enfrentar colectivamente sus situaciones personales. Todas las situaciones constituian problemas de salud severos a las que subyacian condiciones de vida seriamente afectadas. Los diagnósticos de aproximación individuales se dieron en esta sola sesión inicial, de forma muy precisa, multidisciplinaria e integral. El proceso de sanación terapéutica también se inició en esta sesión inicial; los usuarios descubrieron aspectos de resiliencia que no pensaban que aún fueran portadores. En un sistema hospitalario 
institucionalizado esto hubiera tomado posiblemente entre 4 a 6 sesiones distanciadas en el tiempo". (Médico salubrista).

"Estoy convencido de que el taller facilitó la exposición de modo más intenso y rápido de lo que lo hubieran hecho 8 entrevistas individuales clásicas (una para cada una de las 8 personas que asistieron". (Psiquiatra experto en salud mental comunitaria).

"Han encontrado la temporalidad que hace posible la comunicabilidad de asuntos que son complejos. Dos horas parece un tiempo mágico, no puede ser más por agotamiento, no puede ser menos para lograr la confianza y dinámica". (Comunicador social).

"La modalidad propuesta encarna uno de los mejores ejemplos del Cuidado Integral de la Salud por Todos y para Todos. Es una de las mejores experiencias para entender qué es un modelo de Atención Primaria de Salud en el primer nivel, versus un primer nivel hegemónicamente mini hospitalario". (Médico salubrista).

Así, los Grupos de Acogimiento, además de contribuir a la mayor eficacia y eficiencia de la atención, permiten potencialmente la complementariedad y el fortalecimiento continuo de competencias diagnósticas y terapéuticas, reduciendo costos de capacitación. De igual forma, la interacción continua de los profesionales en situaciones concretas de ejercicio profesional, mejora la comunicación, la confianza y fortalece la integración del equipo terapéutico:

\footnotetext{
"Al terminar las sesiones es habitual observar que los profesionales de las diferentes disciplinas siguen comentando los casos y justificando sus posiciones especialmente cuando en algunos puntos ha habido discrepancias, pero se hace en tono amistoso y hasta jocoso. He notado que están contentos y hasta orgullosos de lo que hacen. No es posible eludir una mención al espacio. El CSMC Nancy Reyes ocupa una casa alquilada en una urbanización de la zona. La arquitectura y disposición espacial de la casa alude al espacio doméstico, a la cotidianidad y eso contribuye a facilitar la comunicación entre profesionales $y$ pacientes. No hay un look hospitalario, ni una afectación sanitaria del espacio de atención". (Comunicador social).
}

\section{DISCUSIÓN}

\section{Perspectiva de Salud Mental Comunitaria}

El enfoque de salud mental comunitaria es el eje de la reforma de servicios de Salud Mental en el Perú, tal como se hace explícito en la ley y otros documentos de gestión $(16,17)$. La SMC se basa en el concepto de articulación de saberes profesionales (especializados y generales) y comunitarios para el cuidado de la salud mental. Los Grupos de Acogimiento involucran e integran el punto de vista de los usuarios en el proceso de formulación de los casos y marcan la pauta para la generación de una relación terapéutica más horizontal entre usuarios y profesionales, acercando saberes comunitarios y profesionales en un mayor nivel de paridad que es el derrotero de la SMC $(18,19)$.

Los Grupos de Acogimiento al combinar la confidencialidad colectiva, la participación empoderada de los usuarios, y la legitimación y diálogo abierto de saberes comunes y disciplinarios (20), reduce el riesgo de excesos y omisiones diagnósticas y de abuso de poder cognoscitivo, terapéutico o social. Asimismo, crea las condiciones para el cuidado continuo y colaborativo de la salud mental de las personas, convirtiendo la salud mental de un individuo en un asunto relevante para el otro. Esta es la base del compromiso y la participación en el cuidado comunitario y continuo de la salud mental.

\section{Perspectiva de Salud Pública}

La Salud Pública supone la puesta de recursos y esfuerzos de Estados y sociedades para el cuidado de la salud de las personas, a través de una amplia gama de estrategias y modelos, donde la Atención Primaria de Salud (APS) tiene un lugar preponderante (21). La eficacia, la eficiencia, la pertinencia cultural, la equidad, la participación y el respeto de los derechos de las personas son valores inalienables para la Salud Pública (22), que en el Perú se viene implementando bajo el Modelo de Cuidado Integral de Salud por Curso de Vida para la Persona, Familia y Comunidad. Este modelo es la versión mejorada del Modelo de Atención Integral de Salud Basado en Familia y Comunidad - MAIS BFC que propone el MINSA, y la implementación de las Redes Integrales de Salud (RIS) en el Perú (23).

Los Grupos de Acogimiento se insertan claramente en el principio del autocuidado de la salud, la atención primaria de la salud y la humanización de los servicios 
de salud, ofreciendo herramientas y estrategias muy potentes para el Modelo de Cuidado Integral de Salud y las Redes Integrales de Salud que son los ejes de la política pública de salud en el Perú.

Asimismo, la experiencia de un año de ejecución continua de Grupos de Acogimiento como actividad diagnóstica predominante, y sin contratiempos significativos, evidencia la viabilidad de la implementación de esta práctica y sostenibilidad en los CSMC y su posible escalamiento en otros ámbitos de atención en salud mental con enfoque comunitario.

\section{Perspectiva de Ciencias de la comunicación}

La comunicación, más allá de los actos de producir, transmitir y recibir mensajes es entendida como un flujo de interacciones que va creando y transformando vínculos entre las personas actuantes y adaptando sus subjetividades para consolidar la convivencia coyuntural de un grupo (24). La interacción comunicativa está en la base de todo el proceso de acogimiento donde el discurso confesional e íntimo que se alterna con la escucha activa de todos los participantes se instala como un dispositivo de creación de confianza que diluye barreras generacionales, filiales, profesionales y terapéuticas, en favor de una narrativa permeable a la escucha, la formulación de un discurso estratégico de revelación del sufrimiento personal (25). La dinámica semiestructurada de los grupos de acogimiento conlleva que las narrativas de sufrimiento vayan cambiando de tono a narrativas críticas y de superación instalando un nuevo universo simbólico de la comunicación que va desplazando al universo simbólico de sus escenarios cotidianos. Esto explica el hecho de que en tiempos relativamente breves se logra establecer compromisos y alianzas colaborativas múltiples poniendo de manifiesto el rol de la comunicación en los procesos de enfermar y sanar las mentes y seguramente los cuerpos y la vida de los participantes (26).

\section{Perspectiva de Ciencias sociales}

Las Ciencias Sociales ponen de manifiesto el hecho de que las relaciones de poder o saber entre los individuos obedece principalmente a sus identidades o pertenencias a colectivos o segmentos sociales. El encuentro de dos o más personas es en sí el encuentro de géneros, clases sociales, generaciones, roles y otras identidades colectivas que comparten intereses, ideologías, cosmovisiones, códigos de comunicación, y la intersección de una multiplicidad de identidades y pertenencias que predisponen a alianzas o rivalidades manifiestas o latentes (27).

En el modelo clínico convencional la relación usuario profesional está estructurada de forma asimétrica, en atención a variables o estructuras de género, edad, profesión, modernidad; estableciendo una relación asimétrica abismal que pone al profesional en el papel de sujeto cognoscente y al usuario como sujeto cognoscible (objeto) (28). En los Grupos de Acogimiento se evidencia el empoderamiento progresivo de los usuarios y la relación horizontal, probablemente asociado al hecho de que la metodología hace explícita las identidades compartidas y colectivas entre los participantes, abriendo camino a la expresión de acuerdos o desacuerdos y al establecimiento de alianzas que relativizan los roles estereotipados y la relación de poder asimétrica históricamente estructuradas entre profesionales y usuarios, padres e hijos, hombres y mujeres (29). Esto se ve reforzado por el reconocimiento $\mathrm{y}$ fortalecimiento de su agencia, ya que los usuarios cumplen también el rol de observadores y analizadores de las problemáticas poniendo en situación de diálogo de saberes desde las teorías y las experiencias (30). Esto reduce el exceso del poder terapéutico, el ejercicio de discriminación e incluso los actos de abuso de poder o violencia que son difíciles de auditar o evidenciar en una consulta individual tradicional, precisamente por el poder confesional $(31,32)$.

\section{CONCLUSIONES}

Los Grupos de Acogimiento operacionalizan los principios fundamentales de la Salud Mental Comunitaria, son una herramienta valiosa para la expansión del Modelo de Cuidado Integral como eje de la política pública de salud en el Perú, diluyen las barreras comunicacionales propias de la relación médico-paciente; y acortan las distancias sociales de los participantes posibilitando mejores alianzas terapéuticas y de cuidado.

\section{Correspondencia:}

\author{
Dr. Humberto Castillo-Martell \\ Malecón Paul Harris 396 Dpto 101. Barranco. Lima, \\ Perú. \\ Correo electrónico: walter.castillo@upch.pe
}

Financiamiento: por los propios autores.

Conflictos de interés: los autores declaran no tenerlos. 


\section{REFERENCIAS BIBLIOGRÁFICAS}

1. Castillo-Martell H. Horizontes epistemológicos de la Psiquiatría: Objetivando La subjetividad. Rev Neuropsiquiatr. 2014;77(2):78-85.

2. Fernandez-Liria A. De las psicopatologías críticas a la crítica de la psicopatología. Rev Asoc Esp Neuropsiq. 2001;21(80):57-69.

3. Prvulovic D, Hampel H. ¿Un cambio de paradigma en el diagnóstico psiquiátrico moderno?: Anomalías en las redes neuronales como concepto fisiopatológico y nueva herramienta de diagnóstico. Rev Psiquiatr Salud Ment. 2010;3(4):115-8.

4. Fernandez-Liria A. Algunas reflexiones sobre la práctica pública y la integración de las psicoterapias. Rev de Psicopatol y Psicol Clin. 1999;4(3):203-213.

5. Canut MTL. Enfermería de Salud Mental: El arte y la ciencia del cuidar. Revista Española de Enfermería de Salud Mental. 2018;4(2):1-2.

6. Carrillo A. Profesiones sanitarias y lucha de poderes en el México del Siglo XIX.Asclepio. 1998;50(2):149168.

7. Lolas F. Diagnóstico psiquiátrico: Rótulo, trastorno, comorbilidad. Rev Méd Chile. 2000;128(7):705-707.

8. Carneiro F. A Praxis da ecologia de saberes: Entrevista de Boaventura De Sousa Santos. Tempus, Actas de Saúde Colet. 2014;8(2):331-338.

9. Menéndez E. Las enfermedades ¿Son solo padecimientos?: Biomedicina, formas de atención paralelas y proyectos de poder. Salud Colect. 2015;11(3):301-330

10. Fernandez-Liria A. La nueva actualidad de la salud mental. Rev Asoc Esp Neuropsiq. 2008;28(1):3-5.

11. Sepúlveda R, Ramírez J, Zitko P, Ortiz AM, Norambuena P, Barrera Á, et al. Implementing the Community Mental Health Care Model in a Large Latin-American Urban Area. Int J Ment Health Syst. 2012;41(1):62-72.

12. Valderrama JM. Normalización y biopolítica en la psiquiatría y la salud mental. Cad de Pesquisa Interdisc em Ci-s Hum-s. 2014;15(107):29-48

13. Miranda J, Diez-Canseco F, Araya R, et al. Transitioning Mental Health into Primary Care. Lancet Psychiatry. 2017; 4(2): 90-92.

14. Castillo-Martell H, Cutipé-Cárdenas Y. Implementación, resultados iniciales y sostenibilidad de la reforma de servicios de salud mental en el Perú, 2013-2018. Rev Peru Med Exp Salud Publica. 2019; 36(2): 326-333.

15. Castillo-Martell H, Carrasco-Montoya N, ValdiviaQuintanilla A, Zambrano-Leon C, Perez-Arce L, Pariona-Ruiz E, et al. Grupos de acogimiento: Sistematización de un modelo participativo para la formulación diagnóstica y terapéutica en un centro de salud mental comunitaria. Chorrillos-Perú, 2018. Rev Neuropsiquiatr. 2021;84(1):11-18.

16. Congreso de la República del Perú. Ley 30947: Ley de Salud Mental. Lima: Diario Oficial el Peruano; 30 de abril de 2019

17. Presidencia de la República del Perú. Decreto Supremo que aprueba el Reglamento de la Ley de Salud Mental. Lima: Diario Oficial el Peruano;4 de marzo de 2020.

18. Mata I, Ortiz A. Formulación de casos en salud mental: una guía de entrenamiento. En: Retolaza A, editor. Trastornos mentales comunes: Manual de orientación. Madrid: Asociación Española de Neuropsiquiatría; 2009. pp. 195-213.

19. Ramírez J, Sepúlveda R, Zitko P, Ortiz AM. Consulta de urgencia psiquiátrica y modelo comunitario de atención en salud mental. Rev Chil Salud Pública. 2010;14(1):18-25.

20. Rojas R. Convergencias y diferencias entre el pensamiento complejo y la ecología de saberes. Sophía. 2020;(29):69-91.

21. Carbone-Campoverde F, Flores Y. La atención primaria en salud. La experiencia peruana. Rev Per Ginecol obstet. 2018; 64(3):367-73.

22. Carbone-Campoverde F. Salud pública centrada en la persona, la familia y la comunidad. Rev Peru Med Exp Salud Publica. 2016;33(4):837-838

23. Rivero D, Sánchez T, Ormachea J, CarboneCampoverde F. Cuarenta años después de Alma Ata: la actualidad de sus conceptos fundamentales. Rev Peru Med Exp Salud Publica. 2018;35(4):675-677.

24. Wolton D, Polo M. Salvemos la comunicación: aldea global y cultura. Una defensa de los ideales democráticos y la cohabitación mundial. Barcelona: Editorial Gedisa; 2006.

25. Palacio C. El lenguaje humano no opera como ponemos a conversar a los animales en las fábulas. Una reflexión sobre el lenguajear, el conversar, el dialogar, el informar y el comunicar. Katharsis. 2011;1:51-72.

26. Malet P. Atención y sufrimiento: Una aproximación fenomenológica. Isegoría. 2019;(60):233-252.

27. Duran FE. Las estructuras sociales en el marco de la globalización. Rev int sociol. 2018;58(27):125-150.

28. Paraskeva JM. Justicia contra el epistemicidio: Hacía una breve crítica de la razón occidental moderna. Con-Ciencia Social. 2020;(3):157-174.

29. Beviá B, Girón M. Poder, estigma y coerción: Escenarios para una práctica no autoritaria en salud mental. Rev Asoc Esp Neuropsiq. 2017;37(132): 321-329.

30. Rodríguez J. Proceso relacional de grupo e intersubjetividad: La presencia y la sintonía como factores terapéuticos en psicoterapia de grupo. Rev Psicoterap. 2016;27(105):85-94. 
Análisis del funcionamiento y rol de los Grupos de Acogimiento en la atención de la salud mental comunitaria. Lima, Perú.

31. Desviat M. La reforma psiquiátrica 25 años después de la Ley General de Sanidad. Revista Española de Salud Pública. 2011;85(5):427-436.
32.Foucault M. El poder psiquiátrico. Buenos Aires: Fondo de Cultura Económica, 2005.

Recibido: 14/06/2021

Aceptado: 23/10/2021 